\title{
GENERALIZATION ON UNIQUENESS OF MEROMORPHIC FUNCTIONS OF A CERTAIN DIFFERENTIAL POLYNOMIALS
}

\author{
HARINA P. WAGHAMORE AND HuSNA V.
}

Abstract. In this paper by introducing the notion of multiplicity we study the uniqueness of meromorphic functions concerning differential polynomials and obtain some results. The results of the paper improve and generalize some results due to Jin-Dong Li [7].

Mathematics subject classification (2010): Primary 30D35.

Keywords and phrases: Differential polynomial, Meromorphic function and Entire function.

\section{REFERENCES}

[1] J. Clunie, On a result of Hayman, J. London Math. Soc. 42 (1967), 389-392.

[2] R. S. DYAVANAL, Uniqueness and value-sharing of differential polynomials of meromorphic functions, J. Math. Anal. Appl. 374, (2011) 335-345.

[3] W. K. Hayman, Picard value of meromorphic function and their derivatives,Ann.Math 70 (1959), 9-24.

[4] W. K. Hayman, Meromorphic Functions, Clarendon Press, Oxford, 1964.

[5] I. LAHIRI, Weighted sharing and uniqueness of meromorphic functions, Nagoya Math. J. 161 (2001), 193-206.

[6] J. D. LI, Uniqueness of meromorphic function sharing one value IM, J. Sichuan univ (Nat,Sci,Ed.), 45, 1 (2008), 21-24.

[7] JIN-DONG LI, Notes on the uniqueness and value sharing for meromorphic functions concerning differential polynomials, Ukrainian Mathematical Journal, 66, 10 (2014), 1357-1366.

[8] XiaO-Min Li AND H. X. Yi, Uniqueness of meromorphic functions whose certain nonlinear differential polynomials share a polynomial, Comput. Math. Appl. 62 (2011), 539-550.

[9] C. C. YANG AND X. HUA, Uniqueness and value sharing of meromorphic functions, Ann. Acad. Sci. Fenn. Math. 22, (1997) 395-406.

[10] H. X. YI AND C. C. YAng, Uniqueness Theory of Meromorphic Functions, Sci. Press, Beijing, 1995.

[11] L. YANG, Distribution Theory, Springer-Verlag, Berlin, 1993.

[12] Q.C.ZHANG, Meromorphic function that share one small function with its derivative, J. Inequal Pure and Appl. Math. 6, 4 (2005), Art. 116. 